
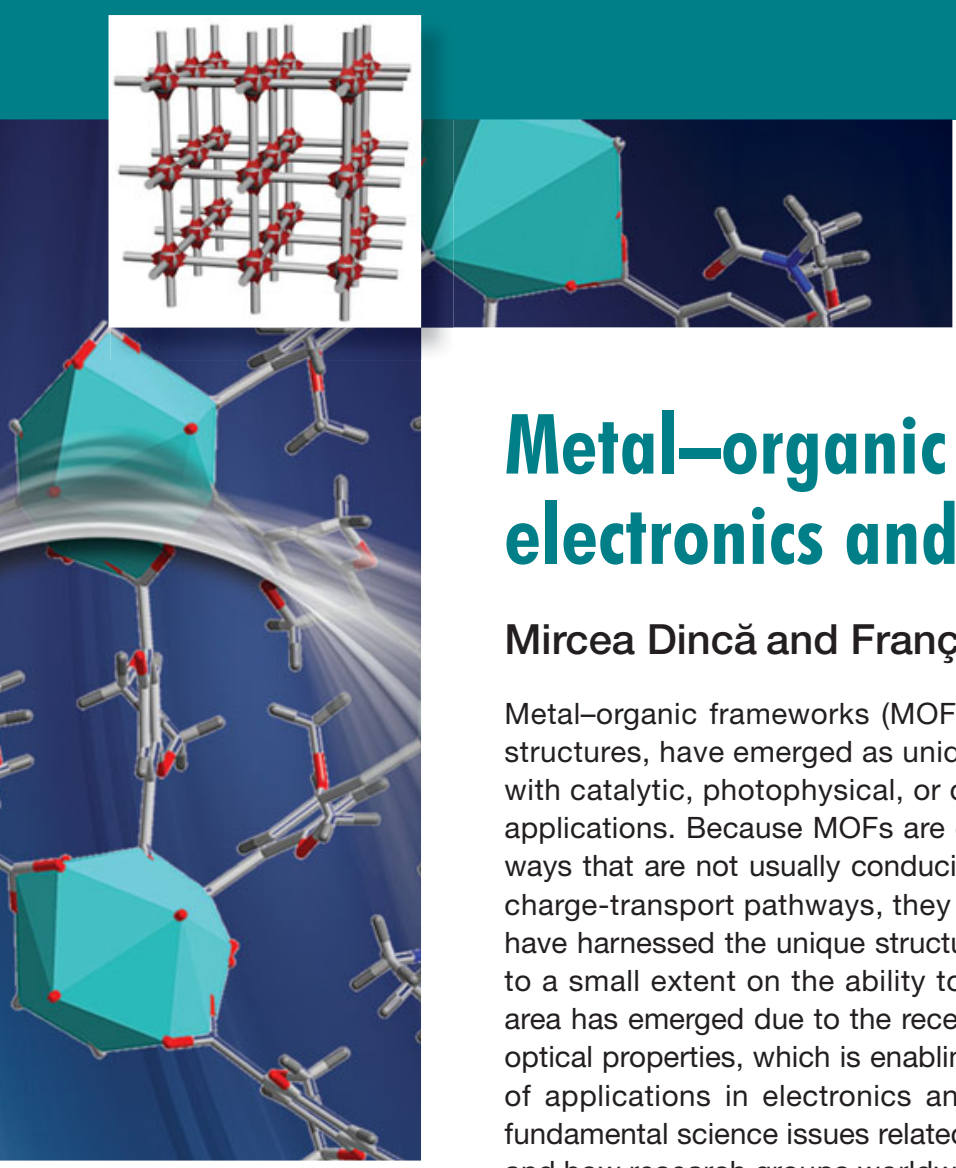

\title{
Metal-organic frameworks for electronics and photonics
}

\author{
Mircea Dincă and François Léonard, Guest Editors
}

\begin{abstract}
Metal-organic frameworks (MOFs), with their crystalline nanoporous three-dimensional structures, have emerged as unique multifunctional materials that combine high porosity with catalytic, photophysical, or other properties to reveal new fundamental science and applications. Because MOFs are composed of organic molecules linking metal centers in ways that are not usually conducive to the formation of free-charge carriers or low-energy charge-transport pathways, they are typically insulators. Accordingly, applications so far have harnessed the unique structural properties and porosity of MOFs, which depend only to a small extent on the ability to manipulate their electronic structure. An exciting new area has emerged due to the recent demonstration of MOFs with controlled electronic and optical properties, which is enabling new fundamental science and opens up the possibility of applications in electronics and photonics. This article presents an overview of the fundamental science issues related to controlling electronic and optical properties of MOFs, and how research groups worldwide have been exploring such properties for electronics, thermoelectrics, photophysics, and charge storage.
\end{abstract}

\section{Metal-organic frameworks}

Metal-organic frameworks (MOFs) ${ }^{1}$ are coordination polymers that exhibit long-range order (i.e., crystallinity) and permanent porosity with pores ranging in size from $0.2 \mathrm{~nm}$ to more than $3 \mathrm{~nm}$, thus spanning the microporous (pores less than $2 \mathrm{~nm}$ ) and mesoporous (pores less than $50 \mathrm{~nm}$ ) regimes. MOFs form by self-assembly processes involving metal ions and organic ligands bearing at least two (often more) metal-binding functional groups (Figure 1). The latter play an essential role because the bond established between the metal ions and the organic ligands is the weakest link in the ensuing material, defining the stability with respect to all outside stimuli: temperature, moisture, and energy input. The most commonly used metal-binding functionalities are carboxylic acids, although other acidic (e.g., azoles, ${ }^{2}$ phosphonic acids ${ }^{3}$ ) and neutral (e.g., pyridines) ligands are becoming prominent, especially in view of the relatively weak bond formed between carboxylates and late first-row transition metals, which comprise the vast majority of existing MOF structures.

Most often, the self-assembly process leads to aggregation of metal ions into secondary-building units (SBUs), which are multinuclear metal clusters with more complex structural and electronic features than single-metal ions. ${ }^{4}$ It is the combination of diverse SBUs and a wide variety of potential organic ligands that has given rise to thousands of MOFs thus far that differ in topology, pore size and shape, and chemical composition. Indeed, MOFs form one of the most diverse classes of materials in existence today, offering tunability at the level of the metal ions, the organic ligands, and the functional groups connecting the two.

The most prominent use of MOFs to date takes advantage of their extreme porosity and the ability to change the polarity, size, shape, and chemical composition of the pore surface. These factors have enabled applications in, for example, gas storage, ${ }^{5}$ in difficult gas separations, ${ }^{6}$ and in catalysis. ${ }^{7-9}$ These prominent applications take advantage of the molecular nature of MOFs, which allow the treatment of solid-substrate interactions locally, on the molecular scale. The utility of MOFs in applications requiring energy or charge transport is limited, however, because the electronic structure of nearly all MOFs show essentially flat bands with minimal band dispersion. Put another way, the electronic states in MOFs are localized and are best described as molecular orbitals rather than delocalized, band-like states. Although the absence of disperse bands need not affect exciton transport or charge hopping, which can enable interesting applications on their own (as highlighted by the articles in this issue), increased band dispersion is critical for long-range charge transport and high-charge mobility, as required for most electronic applications. Recent reports have 


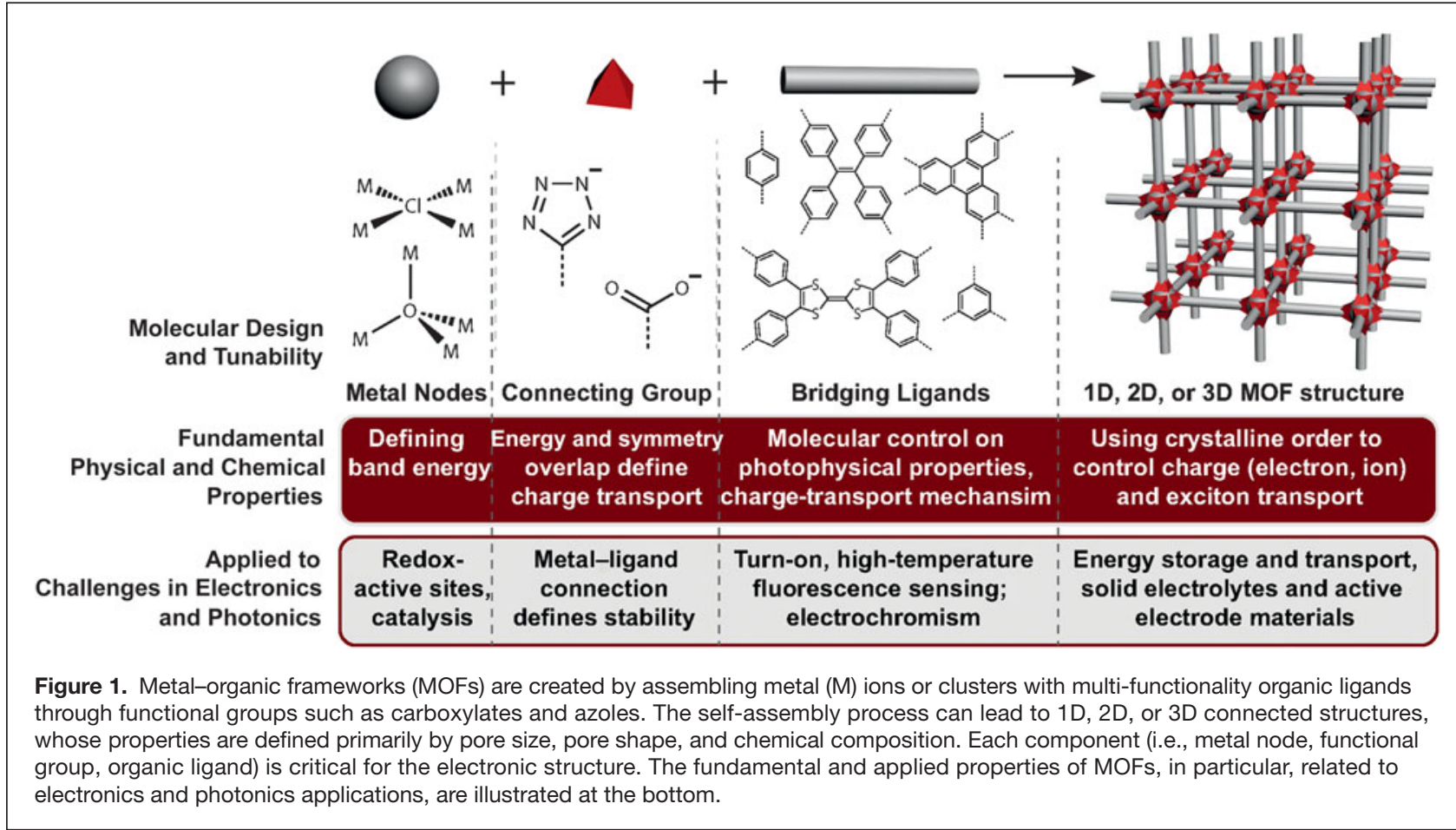

started addressing these basic concepts in band-structure engineering. ${ }^{10,11}$ Consequently, new electronic and photonic MOFs have emerged. ${ }^{12,13}$

\section{Electronic structure of MOFs: Challenges and opportunities in electronics and photonics}

Two primary reasons are arguably the root cause of the insulating nature of MOFs (see the article by Walsh et al. in this issue). They originate from the need to transport charge through the organic linkers and through the ligand-SBU linkages. First, the organic linkers typically have highest occupied molecular orbital-lowest unoccupied molecular orbital (HOMO-LUMO) energy gaps that usually exceed $3 \mathrm{eV}$. This makes it less likely that a metal center will have energy levels that align with those of the ligand. Second, the valence orbitals on the linkers and the SBUs are typically symmetry mismatched. The direct consequence of these factors is that the electronic structure of the MOF crystal is often close to that of the linker (or the metal) alone. This translates to poor extended electronic coupling between the metal center and the linker, which prevents efficient charge transport through the system.

As previously discussed, the resulting electronic bands all have small dispersion, giving large effective masses:

$$
m^{*}=\hbar^{2}\left(\partial^{2} E / \partial k^{2}\right)^{-1},
$$

where $E(k)$ is the energy of a given electronic band as a function of the electronic momentum $k$. This is important because the carrier mobility is inversely proportional to the effective mass:

$$
\mu \propto 1 / m^{*}
$$

and therefore, the band dispersion determines how well the material can carry electrical current. The small band dispersion in MOFs is an indication that electrons are strongly localized rather than delocalized over the whole lattice. Thus, one strategy to impart conductivity is to alter the electronic coupling between the metal centers and the ligands in order to favor delocalization. ${ }^{14}$ Another strategy is to design MOFs that circumvent the issue with the metal-ligand interaction by creating transport paths between linkers. ${ }^{15}$ We note that even if these strategies are successful, other challenges will need to be addressed. For example, it is well known from inorganic and organic semiconductors that controlling charge injection at electrical contacts is essential to realize high-performance devices. ${ }^{16}$ Similarly, learning to control doping (both $n$-type and $p$-type) is essential to realize the building blocks of many technologies (e.g., $p-n$ junctions, Schottky diodes, and complementary transistors).

Although band-like transport is desirable when high mobility is key for a certain application, it is well known from organic semiconductors and molecular conductors that other transport processes besides band transport can lead to reasonable electrical conductivity. ${ }^{17}$ Therefore, it is valuable to also consider the MOF system as composed of electronically localized centers connected by bridges and to consider the conditions under which such a system can be electrically conductive. Extensive work on molecule-bridge-molecule systems has shown that excited-state transport can be considered as "generalized" hopping, whereby configurational reorganization of the bridge can favor dynamic charge transfer between the two molecules. ${ }^{18}$ In fact, simple expressions make the connection between the energetics of the system 
and the mobility through quantum mechanical transition rates. ${ }^{19}$ We anticipate that application of this approach to MOFs could serve as a key technique to identify promising molecular designs.

The realization of electrically conducting MOFs opens a number of new avenues for applications. Some of these are shown in Figure 2, which is representative of the articles discussed in this issue. Conducting MOFs could serve as the basis for new electronic materials (e.g., thin flexible films) ${ }^{20}$ that could solve some of the issues encountered with existing organic materials, such as the difficulty in realizing highperformance $n$-type materials. Further down the line, one could imagine a new field of "crystalline" molecular electronics where the three-dimensional (3D) MOF structure could address the difficulties in positioning single molecules between electrodes. An attractive application that combines electrical conductivity with the nanoporous structure is resistive chemical sensing, whereby targeted molecules to be sensed can infiltrate the MOF pores and change the conductivity. ${ }^{21}$ Along the same lines, the nanoporous structure should also be beneficial for thermoelectrics, ${ }^{22}$ where the nanoporous MOF structure should give inherently low thermal conductivity, and the large number of ligands and metal centers could allow for tuning of the Seebeck coefficient. A similar situation occurs for battery electrodes, ${ }^{23}$ since a conducting MOF can afford high-charge capacity due to the high pore-to-volume ratio.

Finally, conducting MOFs also open new paths for photonic applications, including, but not limited to, solar-energy conversion and light-emitting diodes (LEDs). ${ }^{24}$ One can envision embedding LED emitters and various organic chromophores directly in the MOF pores to induce unique effects such as conversion of photons from low to high energy (upconversion) through triplet-triplet exciton annihilation and the subsequent directional energy transfer that can occur due to the ordered nature of the MOF chemical units. ${ }^{25,26}$

\section{In this issue}

The articles in this issue of MRS Bulletin highlight and detail some of the recent progress in understanding and developing electronic and photonic applications of MOFs. In their article, Leong et al. discuss the different strategies to create MOFs with electronic structures that make them intrinsically conducting. Allendorf et al. discuss in their article an alternative approach to control electronic and optical properties by inserting guest molecules such as tetracynoquinodimethane in the MOF nanopores. The fundamental aspects of these approaches are underpinned by Walsh et al. in their article in this issue. They present the latest advances in $a b$ initio modeling of MOF electronic and optical properties.

Several of the initial applications for electronic and photonic MOFs appear in the context of energy generation and energy storage. Talin et al. present the unique thermoelectric properties of MOFs and discuss recent demonstrations of thermoelectricity in intrinsically conducting MOFs and in guest-infiltrated MOFs. In their article, Zhang and Awaga highlight the use of MOFs in electrochemical systems, particularly as applied to batteries. Finally, Dolgopolova and Shustova summarize progress in controlling and harnessing the photophysics of MOFs.

\section{Summary}

Progress in science and technology often occurs when materials are imbued with new properties. The realization of MOFs with new electronic and photonic properties opens up an entirely new class of materials that combine organic molecules, 3D crystallinity, and optoelectronic properties. These developments elevate MOFs to the level of solid-state semiconductors while also providing entirely different mechanical, structural, and chemical properties. Considering the already large number of existing MOFs, and the chemist's vast synthetic and analytical toolbox, this area should be rich in new 
fundamental science and applications. For example, an understanding of the basic electronic transport properties of MOFs is still in its infancy, and it is not even clear if the standard experimental and theoretical tools applied to solid-state semiconductors are sufficient to unravel these processes. Similarly, the unique properties of MOFs may open entirely new paradigms for electronic and photonic applications. Last, but not least, computational modeling of these complex structures will prove critical for guiding the rapid development of this field.

\section{Acknowledgments}

Work on electronic and photonic properties of MOFs in the Dincă lab has been supported by the DOE through the Center for Excitonics, an Energy Frontier Research Center (Award DE-SC0001088) and a Young Investigator Award (Award DE-SC0006937), both funded by the DOE Office of Science, Office of Basic Energy Sciences. The work at SNL was supported by Sandia's Laboratory-Directed Research and Development Program. Sandia National Laboratories is a multiprogram laboratory managed and operated by Sandia Corporation, a wholly owned subsidiary of Lockheed Martin Corporation, for the US Department of Energy's National Nuclear Security Administration under Contract DE-AC0494AL85000. We thank C. Hendon for assistance with the production of Figure 2.

\section{References}

1. S. Kaskel, Ed., The Chemistry of Metal-Organic Frameworks: Synthesis, Characterization, and Applications (Wiley-VCH, Weinheim, Germany, 2016).

2. J.-P. Zhang, Y.-B. Zhang, J.-B. Lin, X.-M. Chen, Chem. Rev. 112, 1001 (2012). 3. J.M. Taylor, R. Vaidhyanathan, S.S. Iremonger, G.K.H. Shimizu, J. Am. Chem. Soc. 134, 14338 (2012).

4. D.J. Tranchemontagne, J.L. Mendoza-Cortes, M. O'Keeffe, O.M. Yaghi, Chem. Soc. Rev. 38, 1257 (2009)

5. M.P. Suh, H.J. Park, T.K. Prasad, D.-W. Lim, Chem. Rev. 112, 782 (2012).

6. J.-R. Li, J. Sculley, H.-C. Zhou, Chem. Rev. 112, 869 (2012).

7. M. Ranocchiari, J.A.V. Bokhoven, Phys. Chem. Chem. Phys. 13, 6388 (2011).

8. A.H. Chughtai, N. Ahmad, H.A. Younus, A. Laypkov, F. Verpoort, Chem. Soc. Rev. 44, 6804 (2015).

9. E.D. Metzger, C.K. Brozek, R.J. Comito, M. Dincă, ACS Cent. Sci. 2, 148 (2016).

10. H.Q. Pham, T. Mai, N.-N. Pham-Tran, Y. Kawazoe, H. Mizuseki, D. Nguyen-Manh, J. Phys. Chem. C 118, 4567 (2014).

11. S.S. Park, E.R. Hontz, L. Sun, C.H. Hendon, A. Walsh, T.V. Voohis, M. Dincă, J. Am. Chem. Soc. 137, 1774 (2015)

12. L. Sun, M.G. Campbell, M. Dincă, Angew. Chem. Int. Ed. 55, 3566 (2016).

13. T. Neumann, J. Liu, T. Wächter, P. Friederich, F. Symalla, A. Welle, V. Mugnaini, V. Meded, M. Zharnikov, C. Wöll, W. Wenzel, ACS Nano 10, 7085 (2016).

14. L. Sun, T. Miyakai, S. Seki, M. Dincă, J. Am. Chem. Soc. 135, 8185 (2013).

15. A. Talin, A. Centrone, A.C. Ford, M.E. Foster, V. Stavila, P. Haney, R.A. Kinney, V. Szalai, F. El Gabaly, H.P. Yoon, F. Léonard, M.A. Allendorf, Science 343, 66 (2013).

16. F. Léonard, A.A. Talin, Nat. Nanotechnol. 6, 773 (2011).

17. N. Tessler, Y. Preezant, N. Rappaport, Y. Roichman, Adv. Mater. 21, 2741 (2009).

18. B.S. Brunschwig, C. Creutz, N. Sutin, Chem. Soc. Rev. 31, 168 (2002).

19. Y.A. Berlin, G.R. Hutchison, P. Rempala, M.A. Ratner, J. Michl, J. Phys. Chem. A 107, 3970 (2003)

20. O. Shekhah, J. Liu, R.A. Fischer, C. Wöll, Chem. Soc. Rev. 40, 1081 (2011). 21. M.G. Campbell, D. Sheberla, S.F. Liu, T.M. Swager, M. Dincă, Angew. Chem. Int. Ed. 54, 4349 (2015).

22. K.J. Erickson, F. Léonard, V. Stavila, M.E. Foster, C.D. Spataru, R.E. Jones, B.M. Foley, P.E. Hopkins, M.D. Allendorf, A.A. Talin, Adv. Mater. 27, 3453 (2015). 23. C. Combelles, M.B. Yahia, L. Pedesseau, M.L. Doublet, J. Phys. Chem. C 114, 9518 (2010)

24. X. Zhang, M. Hejazi, S.J. Thiagaraian, W.R. Woerner, D. Banerjee, T.J. Emge, W. Xu, S.J. Teat, Q. Gong, A. Safari, R. Yang, J.B. Parise, J. Li, J. Am. Chem. Soc. 135, 17401 (2013)
25. S. Jin, H.-J. Son, O.K. Farha, G.P. Wiederrecht, J.T. Hupp, J. Am. Chem. Soc. 135, 955 (2013).

26. P. Mahato, A. Monguzzi, N. Yanai, T. Yamada, N. Kimizuka, Nat. Mater. 14, $924(2015)$

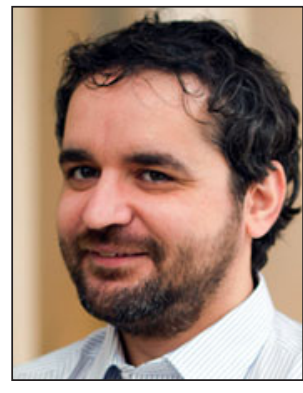

Mircea Dincă is an associate professor of chemistry at the Massachusetts Institute of Technology (MIT). He received his BA degree in chemistry from Princeton University in 2003 and his $\mathrm{PhD}$ degree in inorganic chemistry from the University of California, Berkeley, in 2008. He began his independent career in 2010 after a postdoctoral appointment, also at MIT. Dinca 's research group focuses on the synthesis of new, modular, functional materials for applications in renewable energy and heterogeneous catalysis.

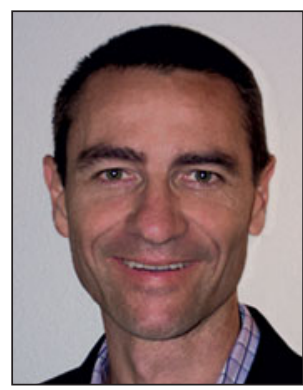

François Léonard is a distinguished member of the technical staff at Sandia National Laboratories. He received his BSC degree in physics from McGill University, Canada, in 1994, and PhD degree in physics from the University of Toronto, Canada, in 1998. He completed postdoctoral research at the IBM T.J. Watson Research Center before joining Sandia in 2000. Leonard's research interests include nanoelectronics and nanophotonics, with a focus on combining experiment and theory to understand the fundamental properties of nanomaterials and their use in devices.

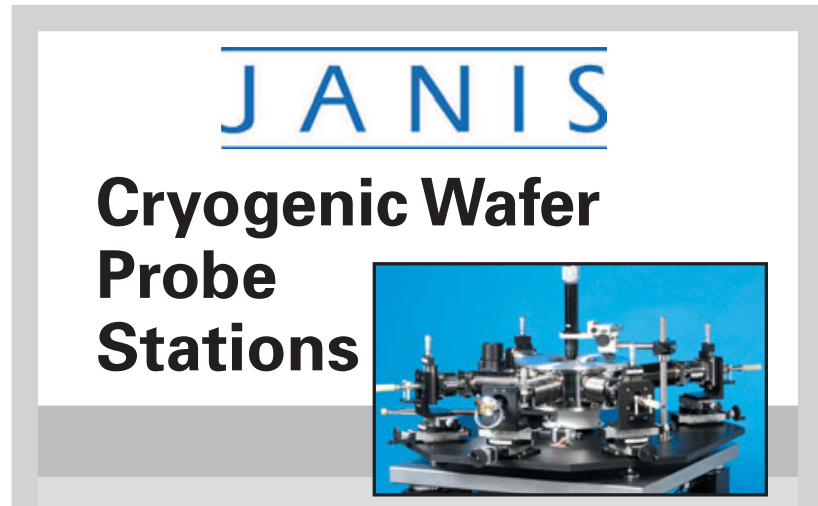 \\ Applications include nano science, materials and spintronics \\ $3.2 \mathrm{~K}-675 \mathrm{~K}$; high vacuum or UHV \\ Up to 8 probes, DC to $67 \mathrm{GHz}$, plus fiber optics \\ Zoom optics with camera and monitor \\ - Cooling options: liquid helium, liquid nitrogen or cryogen free \\ - Horizontal, vertical or vector magnetic field options are available \\ Contact us today: sales@janis.com \\ www.janis.com/ProbeStations.aspx www.facebook.com/JanisResearch

